\title{
Transoesophageal echocardiography reduces invasiveness of cavoatrial tumour thrombectomy
}

\author{
Robert Sobczyński ${ }^{1}$, Tomasz Golabek ${ }^{2}$, Piotr Mazur ${ }^{1,3}$, Piotr Chłosta ${ }^{2}$ \\ ${ }^{1}$ Klinika Chirurgii Serca, Naczyń i Transplantologii, Institute of Cardiology, Jagiellonian University Medical College, Krakow, Poland \\ ${ }^{2}$ Department of Urology, Jagiellonian University Medical College, Krakow, Poland \\ ${ }^{3}$ Institute of Cardiology, Jagiellonian University Medical College, Krakow, Poland
}

Videosurgery Miniinv (e-pub, ahead of print) DOI: $10.5114 /$ wiitm.2014.44281

\begin{abstract}
The traditional approach to cavoatrial thrombus excision requires median sternotomy, cardiopulmonary bypass with or without hypothermia and circulatory arrest and is associated with significant morbidity and mortality. We describe a transoesophageal echocardiography guided balloon catheter assisted technique for cavoatrial thrombectomy that avoids thoracotomy, extracorporeal circulation and circulatory arrest as an alternative to traditional methods. A 74-year-old man presented with a right solid renal mass confined to the kidney with thrombus extension through the right renal vein and the inferior vena cava into the right atrium. A right radical nephrectomy with cavoatrial thrombectomy under transoesophageal echocardiography guidance was successfully achieved using a balloon catheter-assisted technique with minimal intra-and postoperative morbidity. Cavoatrial tumour thrombectomy can be successfully performed without cardiopulmonary bypass, hypothermia and circulatory arrest.
\end{abstract}

Key words: cavoatrial thrombus, renal cancer, thrombectomy, surgical management.

\section{Introduction}

Approximately $4-10 \%$ of renal cell carcinoma (RCC) cases are complicated by the presence of tumour thrombus (TT) in the inferior vena cava (IVC) [1]. Although the extension of thrombus is limited to the infra-diaphragmatic segment of the IVC in most cases, it can reach the right atrium in up to $1 \%$ of patients [2]. Treatment of advanced renal tumours still represents a great surgical challenge [3]. The presence of renal cancer with thrombus is associated with venous congestion, distal embolism and the development of neovascularization, as well as collaterals that form as a result of the inferior vena cava occlusion and, in consequence, increase of the technical difficulty of surgery. The operative treatment depends on the level of thrombus and the extent of thrombus propagation throughout the IVC. In cases with TT of low volume, simple thrombus excision, IVC endoluminal occlusion and thrombus stripping with a venous catheter, minimal access technique and laparoscopic approaches all have been used [4-6]. The traditional approach to cavoatrial thrombus excision requires median sternotomy, cardiopulmonary bypass with or without hypothermia and circulatory arrest, and is associated with significant morbidity and mortality $[1,7]$.

\section{Aim \\ We describe a transoesophageal echocardiog- raphy (TOE) guided balloon catheter-assisted tech- nique for cavoatrial thrombectomy not requiring thoracotomy, extracorporeal circulation and circu- latory arrest as an alternative to the traditional ap- proach.}

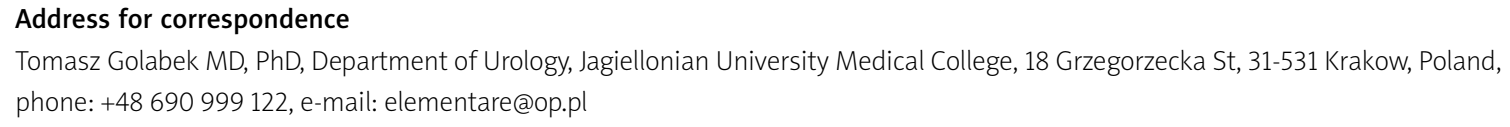

